\title{
Evaluación de la susceptibilidad de especies de Candida al fluconazol por el método de difusión de disco
}

Catalina de Bedout ${ }^{1}$, Julio Ayabaca ${ }^{2}$, Ricardo Vega ${ }^{3}$, Matilde Méndez ${ }^{3}$, Áxel R. Santiago ${ }^{4}$, María Lucrecia Pabón ${ }^{2}$, Angela Tabares ${ }^{1}$, Myrtha Arango ${ }^{1,5}$, Angela Restrepo ${ }^{1}$, Vance Newell

${ }^{1}$ Corporación para Investigaciones Biológicas (CIB), Medellín, Colombia.

2 Hospital de las Fuerzas Armadas, Quito, Ecuador.

${ }^{3}$ Hospital Militar, Bogotá, D.C., Colombia.

${ }^{4}$ Hospital Universitario de Caracas, Caracas, Venezuela.

${ }^{5}$ Universidad de Antioquia, Medellín, Colombia.

${ }^{6}$ Giles Scientific Inc., Santa Barbara, California, USA.

Las infecciones causadas por levaduras del género Candida han aumentado en forma dramática en las últimas décadas, especialmente a nivel hospitalario. Paralelamente con este aumento, se ha notado la aparición de resistencia a los antimicóticos, así como la selección de especies diferentes a Candida albicans, todo lo cual ha hecho necesaria la estandarización de pruebas de susceptibilidad in vitro. Entre las varias pruebas disponibles está la de difusión en agar con discos impregnados de antifúngicos, cuya lectura se hace electrónicamente (BIOMIC), lo que proporciona indicaciones objetivas de la concentración inhibitoria mínima (CIM). Se realizó el estudio para determinar la sensibilidad in vitro al fluconazol de 2.139 aislamientos de especies de Candida provenientes de pacientes atendidos en consulta externa o en salas de hospitalización de centros asistenciales en Colombia, Ecuador y Venezuela (región CELA). Candida albicans fue el microorganismo más frecuentemente aislado (62\%), seguido a distancia por Candida parapsilosis (11\%), Candida tropicalis $(8,5 \%)$, Candida glabrata $(3,5 \%)$ y Candida krusei $(2,2 \%)$. La CIM demostró que el $88,1 \%$ de estos aislamientos eran susceptibles al fluconazol, $5,1 \%$ eran susceptibles-dosisdependiente y $6,8 \%$ resistentes. El $92,1 \%$ de la especie $C$. albicans fue susceptible mientras que la resistencia al fluconazol fue más notoria en las restantes especies. EI BIOMIC es un método rápido y sencillo para la vigilancia epidemiológica de resistencia en levaduras del género Candida recuperadas de pacientes atendidos en centros hospitalarios.

Palabras clave: candidiasis, Candida, resistencia anfúngicos, fluconazol, prueba de disco.

Resistance to fluconazole by Candida species detected using the disk diffusion method.

Infections caused by yeasts belonging to the genus Candida have increased dramatically in the last decades, especially in hospital settings. Concomittantly, antimycotic resistance has emerged, as well as the appearance of non-Candida albicans isolates. To standardize in vitro antifungal susceptibility tests, the agar diffusion test was developed using disks impregnated with the antimycotic compound. Electronic recording of the inhibition zone (BIOMIC), furnishes objective values for the minimal inhibitory concentration (MIC). The fluconazole susceptibility patterns were determined for Candida species isolated from 2.139 patients seen in outpatient clinics or in health-care centers in Colombia, Ecuador and Venezuela. Candida albicans was the species most frequently isolated $(62 \%)$, followed at a distance by Candida parapsilosis $(11 \%)$, Candida tropicalis (8.5\%), Candida glabata $(3.5 \%)$ and Candida krusei $(2.2 \%)$. MIC determinations showed that $88.1 \%$ of these isolates were susceptible to fluconazole, $5.1 \%$ were susceptible-dose-dependant and $6.8 \%$ resistant. An important proportion $(92.1 \%)$ of the $C$. albicans isolates proved susceptible while resistance predominated in the remaining species. These results indicate that the BIOMIC method is rapid and simple, constituting a suitable tool for the epidemiologic surveillance of resistance in Candida species.

Key words: candidiasis, Candida, antifungals, resistance, fluconazole, disk diffusion. 
Las infecciones causadas por levaduras del género Candida han aumentado en forma dramática en las últimas décadas como consecuencia de los progresos de la medicina moderna, la que ha permitido la supervivencia de pacientes en estado crítico, las cirugías de avanzada y tratamientos con compuestos inmunosupresores y citotóxicos, a la par que con antibióticos de amplio espectro. Igual repercusión ha tenido la pandemia del síndrome de inmunodeficiencia adquirida, lo que ha favorecido la aparición de las micosis, convirtiéndolas en procesos diseminados serios o en formas crónicas refractarias al tratamiento (1-3).

Hasta hace unos pocos años, se consideraba que los hongos eran regularmente susceptibles a los antimicóticos; se había observado solamente que Candida spp. podía adquirir resistencia a la 5 flurocitocina (4), anotándose un poco más tarde la aparición de resistencia de $C$. albicans en pacientes con candidiasis granulomatosa crónica bajo tratamiento prolongado con ketoconazol (5). Hasta ese momento, el problema no parecía tener mayor repercusión, pero una década más tarde la situación cambiaría drásticamente al observarse un aumento en la frecuencia de candidemias debidas no sólo a $C$. albicans resistentes sino también a especies diferentes a $C$. albicans, usualmente menos susceptibles a los antimicóticos. Entre estas últimas, se destacaban $C$. krusei, que es intrínsecamente resistente al fluconazol, y $C$. glabrata, cuya susceptibilidad a los azoles es muy variable (6-8). Pero tal vez más notorias fueron las fallas observadas a partir de 1985 en pacientes con sida y mucositis candidiásica bajo tratamiento con fluconazol, quienes desarrollaban candidiasis orofaríngea crónica refractaria a la terapia (9-11). Esta resistencia fue comprobada por las altas concentraciones inhibitorias mínimas (CIM) de los correspondientes aislamientos (12-15).

\footnotetext{
Correspondencia:

Angela Restrepo M., Corporación para Investigaciones Biológicas (CIB), Carrera 72 A\# 78 B-141, Medellín, Colombia. Tel: (574) 441 0855; Fax: (574) 4415514. angelares@geo.net.co
}

Recibido: 05/11/02; aceptado: 07/02/03
El presente estudio tuvo como objetivo determinar la resistencia al fluconazol de las especies de Candida provenientes de pacientes residentes en Colombia, Ecuador y Venezuela, región designada CELA. Este trabajo hace parte de un estudio global de vigilancia antimicótica en el que participan 48 instituciones de 33 países (16).

\section{Materiales y métodos}

La muestra sometida a estudio estuvo representada por 2.139 aislamientos de Candida spp. que fueron obtenidos durante el periodo comprendido entre junio de1997 y diciembre de 2001 , procedentes de pacientes con procesos clínicos significativos que recibían atención en los servicios de dermatología, medicina interna, unidad de cuidados intensivos, cirugía, ginecología y obstetricia, urología, hematología y otros no clasificados. Las levaduras fueron aisladas de diferente muestras clínicas provenientes de tracto genitourinario, respiratorio y gastrointestinal, piel y tejidos blandos, SNC, sangre y otros (detritos ungueales y muestras no clasificadas). Todos los aislamientos se identificaron a género y a especie por los métodos usuales en cada institución (producción de tubo germinal, de clamidosporas, pruebas de asimilación API 20C Aux (Biomerieux, Marcy.L'Etoile, France) y MicroScan (Rochen Biocare, USA).

Para la determinación de la susceptibilidad al fluconazol se utilizó el método de difusión de disco descrito por Mies et al. (17); este método utiliza sensidiscos de $25 \mu \mathrm{g} / \mathrm{ml}$ de fluconazol (Beckton Dickinson, Spark, MD), agar Mueller Hinton (BBL, Cockeysville, MD) suplementado con glucosa al $2 \%$ y con $0,5 \mu \mathrm{g} / \mathrm{ml}$ de azul de metileno.

Se preparó una suspensión de las levaduras, ajustando su concentración a la turbidez 0,5 de la escala de McFarland con agua destilada estéril. Las cajas con el agar Mueller Hinton suplementado se inocularon con un escobillón humedecido en esta suspensión, de acuerdo con las recomendaciones para las pruebas de difusión de disco para bacterias dadas por el Comité Nacional para los Estándares en el Laboratorio Clínico, NCCLS (National Committee for Clinical and Laboratory Standards, Estados Unidos) (documento M2-A6, 1997) (18) y con incubación 
a $35^{\circ} \mathrm{C}$ durante 18 a 24 horas. La lectura se efectuó en el BIOMIC, un lector de caja de Petri, con un analizador digital de imagen, el cual mide los milímetros de inhibición, los convierte a CIM gracias a una curva de regresión, para luego almacenar los datos electrónicamente (Giles Scientific, 1999, Santa Barbara, CA). Con estos valores y por análisis de regresión se calcularon la CIM 50 y 90 (14).

La interpretación de la CIM para el fluconazol se basó en el diámetro de inhibición, el cual se correlaciona con los puntos de corte recomendados en el método de referencia M27 A de la NCCLS (19-21). Se consideraron como susceptibles los aislamientos cuyas CIM eran menores o iguales a $8 \mu \mathrm{g} / \mathrm{ml}(>19 \mathrm{~mm}$ de inhibición), como susceptibles dosis dependientes (SDD) aquéllos en los que la CIM estaba entre $16-32 \mu \mathrm{g} / \mathrm{ml}$ (13-18 mm de inhibición) y como resistentes aquéllos en que la CIM era igual o superior a $64 \mu \mathrm{g} / \mathrm{ml}$ (0-12 mm de inhibición).

Los controles de calidad se hicieron semanalmente utilizando dos cepas control obtenidas de la ATCC, C. albicans 90028 , la cual presenta un rango de inhibición de 32 a $43 \mathrm{~mm}$, y C. parapsilosis 22019, que muestra un rango de 26 a $37 \mathrm{~mm}$.

Los resultados de la zona de inhibición, la susceptibilidad, el valor de la CIM y el control de calidad fueron almacenados electrónicamente. Los datos obtenidos por los laboratorios participantes fueron enviados periódicamente para análisis, mediante una transferencia de datos, a Giles Scientific (Santa Barbara, CA). En esta transferencia se omite el nombre del paciente y del médico tratante, al igual que aquellos resultados que en un período de 7 días consecutivos correspondieran al mismo paciente y al mismo tipo de aislamiento, así como aquéllos que no estuviesen controlados adecuadamente en el marco de las normas de la NCCLS.

\section{Resultados}

Durante el período de estudio se obtuvieron 2.139 aislamientos de Candida spp., cuya clasificación reveló que Candida albicans, con 1.328 aislamientos (62\%), era la especie más frecuentemente aislada, seguida a distancia por Candida parapsilosis (11\%), Candida tropicalis (8,5\%), Candida glabrata (3,5\%) y Candida krusei $(2,2 \%)$. El grupo denominado Candida spp. con $273(12,8 \%)$ aislamientos, incluyó tanto especies aisladas infrecuentemente $(n=132)$ como aquéllas para las que no se logró determinar la especie $(n=141)$. En conjunto, las especies distintas a $C$. albicans representaron $38 \%$ de todos los aislamientos (cuadro 1).

La mayoría de los aislamientos, $1.885(88,1 \%)$, fueron susceptibles (S) al fluconazol, $110(5,1 \%)$ mostraron ser susceptibles dosis dependiente,

Cuadro 1. Susceptibilidad al fluconazol en los aislamientos de Candida spp, región CELA.

\begin{tabular}{|c|c|c|c|c|c|c|c|c|}
\hline \multirow[t]{3}{*}{ Aislamientos* } & \multicolumn{6}{|c|}{ Susceptibilidad } & & \\
\hline & \multicolumn{2}{|c|}{ Susceptibles } & \multicolumn{2}{|c|}{$\begin{array}{c}\text { Susceptibles dosis } \\
\text { dependientes }\end{array}$} & \multicolumn{2}{|c|}{ Resistentes } & \multicolumn{2}{|c|}{ Total } \\
\hline & $\mathbf{n}$ & $\%$ & $\mathbf{n}$ & $\%$ & $\mathbf{n}$ & $\%$ & $\mathbf{n}$ & $\%$ \\
\hline C. albicans & 1223 & 92,1 & 44 & 3,3 & 61 & 4,6 & 1.328 & 62,0 \\
\hline C. parapsilosis & 206 & 87,7 & 16 & 6,8 & 13 & 5,5 & 235 & 11,0 \\
\hline C. tropicalis & 163 & 90,0 & 10 & 5,5 & 8 & 4,5 & 181 & 8,5 \\
\hline C. glabrata & 53 & 71,6 & 8 & 10,8 & 13 & 17,6 & 74 & 3,5 \\
\hline C. krusei & 33 & 68,8 & 5 & 10,4 & 10 & 20,8 & 48 & 2,2 \\
\hline Candida spp** & 207 & 75,8 & 27 & 9,9 & 39 & 14,3 & 273 & 12,8 \\
\hline Total & 1885 & 88,1 & 110 & 5,1 & 144 & 6,8 & 2.139 & 100,0 \\
\hline
\end{tabular}

* Provenientes de cuatro instituciones (junio1997-diciembre 2001)

** En Candida spp están incluidos 273 aislamientos, 132 clasificados como sigue: C. guilliermondii (4,2\%), C. famata(0,6\%), C. Iusitaniae (0,5\%), C. kefir (0,09\%), C. zelanoides (0,2\%), C. lipolitica $(0,09 \%)$, C. novergensis $(0,09 \%)$, C. pelliculosa $(0,04 \%)$, Pichia spp $(0,1 \%)$; las restantes 141 no fue posible clasificarlas. 
mientras que $144(6,8 \%)$ fueron resistentes $(R)$ (cuadro 1). Al analizar los datos se encontró que $92,1 \%$ de los aislamientos de C. albicans eran susceptibles al fluconazol. Ligeramente menos susceptible resultaron ser $C$. tropicalis $(90 \%)$ y C. parapsilosis (87,7\%). La menor susceptibilidad se encontró en las especies $C$. glabrata y $C$. krusei, con $71,6 \%$ y $68,8 \%$, respectivamente, lo cual indicó que en ellas estaba la mayor resistencia (cuadro 1). Los centros con el mayor número de $C$. albicans resistentes al fluconazol fueron el Hospital Militar de Ecuador con 37/512 $(7,2 \%)$ y el Hospital Universitario de Caracas, Venezuela, con 16/272 (5,9\%).

Al construir un gráfico basado en los datos del diámetro de la inhibición del crecimiento de $C$. albicans y su frecuencia, se observó una curva unimodal en forma de campana (figura 1); para $C$. parapsilosis y $C$. tropicalis se obtuvieron gráficas similares. C. krusei y C. glabrata mostraron mayor dispersión, con un número importante de aislamientos en las categorías S, SDD y R (datos no ilustrados).

Los valores correspondientes a la CIM 90 (cuadro 2 ), señalaron que el $90 \%$ de las especies no albicans, tales como C. glabrata, C. krusei, Candida guilliermondii, Candida famata y Candida zeylanoides presentaron valores $>165 \mu \mathrm{g} / \mathrm{mL}$, por lo que se consideraron resistentes. Por el contrario, cuando se consideraron los aislamientos de C. albicans, los valores de la CIM 50 mostraron

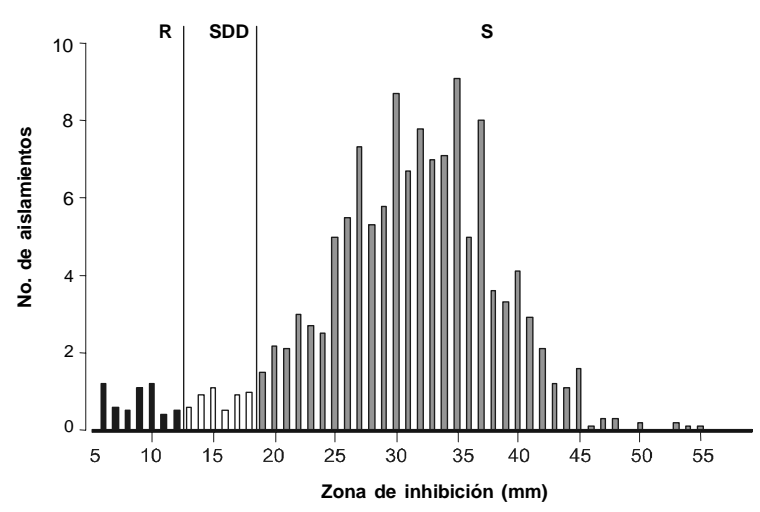

Figura 1. Zonas de inhibición del crecimiento de aislamientos de Candida albicans al fluconazol.

Región CELA: aislamientos provenientes de cuatro instituciones (junio 1997 - diciembre 2001).
Cuadro 2. MIC 50 y MIC 90 del fluconazol para los aislamientos de levaduras provenientes de la región CELA.

\begin{tabular}{|c|c|c|c|c|}
\hline \multirow[t]{2}{*}{ Organismo } & \multicolumn{2}{|c|}{ MIC $(\mu \mathrm{g} / \mathrm{ml})$} & \multirow[b]{2}{*}{$\mathbf{n}$} & \multirow[b]{2}{*}{$\%$} \\
\hline & MIC 50 & MIC 90 & & \\
\hline C. albicans & 1,1 & 17,4 & 1.328 & 62 \\
\hline C. parapsilosis & 3,0 & 32,8 & 235 & 11 \\
\hline C. tropicalis & 2,4 & 25,6 & 181 & 8,5 \\
\hline C. glabrata & 10,6 & $>165$ & 74 & 3,5 \\
\hline C. krusei & 3,9 & $>165$ & 48 & 2,2 \\
\hline C. guilliermondii & 10,6 & $>165$ & 91 & 4,2 \\
\hline C. famata & 4,7 & $>65$ & 14 & 0,6 \\
\hline C. Iusitaniae & 14 & 146,8 & 12 & 0,5 \\
\hline C. zeylanoides & 10,6 & $>165$ & 5 & 0,2 \\
\hline C. kefyr & 83,7 & $>165$ & 2 & 0,09 \\
\hline C. lipolitica & 13,7 & 22,4 & 2 & 0,09 \\
\hline C. norvegensis & 3,7 & 5 & 2 & 0.09 \\
\hline C. pelliculosa & $<0,25$ & $<0,25$ & 1 & 0,04 \\
\hline Pichia spp.* & 47,3 & 60,8 & 3 & 0,1 \\
\hline Candida sp & 1,4 & 47,3 & 141 & 6,6 \\
\hline Total & & & 2.139 & 100 \\
\hline
\end{tabular}

Pichia spp.: fase sexuada de C. pelliculosa o de $C$. guilliermondii.

que eran $\mathrm{S}(1,2 \mu \mathrm{g} / \mathrm{mL})$; no obstante, los valores de la CIM $90(17,4 \mu \mathrm{g} / \mathrm{mL})$ ubicaron estos mismos aislamientos en el rango de SDD.

De acuerdo con las muestras clínicas, $C$. albicans fue la especie más frecuentemente aislada de hemocultivos (40\%), seguida por $C$. tropicalis (18,3\%); el tercer lugar lo compartieron C. glabrata y C. parapsilosis (6,6\%). Al dejar de lado el grupo de muestras clínicas clasificadas como 'otros', los especímenes que tuvieron el número mayor de aislamientos fueron los procedentes de los tractos respiratorio y urinario $15 \%$ y $12,6 \%$, respectiva-mente. En referencia a los servicios hospitalarios con mayor número de muestras positivas, éstos fueron los de dermatología con 493 aislamientos (22,8\%), los de medicina interna con $471(21,7 \%)$ y los de unidades de cuidados intensivos con $312(14,4 \%)$ aislamientos.

\section{Discusión}

El incremento en la frecuencia de infecciones causadas por levaduras, el aumento en la mortalidad de los pacientes afectados y la emergencia de aislamientos resistentes han creado la necesidad de efectuar una vigilancia epidemiológica cuidadosa sobre los aislamientos 
procedentes del medio hospitalario. Se hace ahora indispensable clasificar todas las levaduras del género Candida a nivel de especie $(1,6,15,22$,$) .$ Igualmente importante es la realización de las pruebas de susceptibilidad a los antimicóticos $(7,14,20,23,24)$.

EI NCCLS de los Estados Unidos ha presentado un método de referencia (M27 A, 1997, Villanova, PA) (19) para determinar la susceptibilidad in vitro de las levaduras, el cual es una prueba cuantitativa que se realiza en medio líquido. Este método, sin embargo, se practica sólo en laboratorios especializados que cuentan con personal capacitado. Por ello, se ha desarrollado un método más sencillo, como es el método de difusión de disco (20), el que, a su vez, ha sido modificado para lectura en un sistema computarizado $(13,16,21)$ que lo hace más preciso y reproducible.

Este estudio en la región CELA reveló que $C$. albicans es la especie más frecuentemente aislada $(61,3 \%)$ de muestras clínicas y, especialmente, de hemocultivos $(40 \%)$, como ocurre en otras regiones del mundo $(1,2,7,22,24)$. No obstante, la observación en la región CELA de especies diferentes, las que en conjunto ocupan ya un lugar prominente (38\%) entre los aislamientos clínicos, revela y confirma el incremento de patógenos hospitalarios emergentes como ha sido ya anotado en otros países $(1,6,7,14,15,22,24)$. A pesar de que los aislamientos correspondientes a las especies más resistentes al fluconazol, como son C. krusei y $C$. glabrata, fueron relativamente infrecuentes, $2,2 \%$ y $3,5 \%$, respectivamente, existe el peligro de que aumenten en el futuro, lo que señala la necesidad de estudios periódicos de vigilancia. Liebowitz et al. (16) mostraron una disminución significativa $(p>0,0001)$ de $C$. albicans al comparar dos años de estudio, $69,9 \%$ en 1998 y $67,3 \%$ en 1999 , con el incremento subsiguiente de las especies diferentes a $C$. albicans.

En el presente trabajo, las pruebas de susceptibilidad al fluconazol revelaron que durante el período del estudio, $88,1 \%$ de los aislamientos de Candida eran susceptibles, $5,1 \%$ SDD y $6,8 \%$ resistentes. C. albicans mostró alta susceptibilidad
$(92,1 \%)$ a este triazol, observación que fuera corroborada por la CIM 50 cuyo valor fue de 1,1 $\mu \mathrm{g} / \mathrm{ml}$, mientras que los valores de la CIM $90(17,4$ $\mu \mathrm{g} / \mathrm{ml}$ ) las catalogaban ya como SDD. Las cifras más altas de resistencia fueron observadas en las especies diferentes a $C$. albicans, tales como C. glabrata y C. krusei con $17,6 \%$ y $20,8 \%$ de aislamientos resistentes, respectivamente. Tales hallazgos fueron respaldados por la CIM 90 (>165 $\mu \mathrm{g} / \mathrm{ml}$ ). Igual sucedió con otras especies que, aunque menos frecuentes, presentaron una baja cobertura en relación con la CIM 90. Los hallazgos anteriores están de acuerdo con lo informado en la literatura $(7,8,14-17,22,24)$.

Si bien $C$. albicans fue la especie más frecuentemente aislada de hemocultivos (40\%), otras especies como C. tropicalis, C. glabrata y C. parapsilosis representaron en conjunto $31,6 \%$ de todos los aislamientos. En otros estudios se ha informado que $C$. tropicalis es la más frecuente de las especies no albicans aislada de hemocultivos $(14,26,27)$. En el estudio del grupo SENTRY (7), que incluyó cultivos de Suramérica, la frecuencia de las especies aisladas fue similar a la informada en este estudio en lo que concierne a $C$. albicans $(40,5 \%)$, pero con variaciones en referencia a C. parapsilosis $(38,1 \%)$, C. tropicalis $11,9 \%$, C. glabrata y C. guilliermondii, $2,4 \%$ cada una, y otras especies, $4,7 \%$.

Los especímenes clínicos de los cuales se obtuvo un mayor número de aislamientos fueron los del tracto respiratorio, con 326 (15\%), y urinario, con $272(12,6 \%)$, lo que indica que la colonización por Candida en los servicios hospitalarios reviste importancia y debería ser objeto de atención, aplicando para ello medidas relacionadas con el empleo racional de las terapias a base de antibióticos de alto espectro o a las terapias empleadas en forma prolongada $(3,6,14,15,23)$. Igualmente, deberían vigilarse la contaminación dentro de los servicios hospitalarios y el uso de procedimientos invasores en la práctica médica $(2,15,25,26)$.

Es importante resaltar que la colonización por Candida en pacientes de alto riesgo predice una posible candidiasis hematógena $(14,22,26)$. Los servicios hospitalarios con mayor número de 
muestras positivas fueron los de dermatología con $493(22,8 \%)$, medicina interna con 471 aislamientos $(21,7 \%)$, y la unidades de cuidados intensivos con 312 aislamientos (14,4\%), siendo los servicios de medicina interna y las unidades de cuidados intensivos los lugares donde se encuentran los pacientes con mayor riesgo de colonización, quienes pueden desarrollar candidiasis invasora, ya que tienen varios factores predisponentes $(14,26,27)$.

En forma global y en la región CELA, la resistencia al fluconazol de las levaduras del género Candida fue baja (6,8\%); se contabilizaron, además, $5,1 \%$ de aislamientos SDD. EI BIOMIC es muy rápido, sencillo y económico. Estas circunstancias, unidas a la precisión de sus lecturas, lo convierten en una ayuda valiosa, capaz de guiar la terapia en forma más eficaz, pues proporciona, además, los datos requeridos para la vigilancia epidemiológica de la resistencia y del predominio de las levaduras en hospitales $(14,22,25)$. Estas medidas contribuirán al mejor y más oportuno control de las afecciones producidas por las especies del género Candida.

\section{Agradecimientos}

Este estudio fue patrocinado por Pfizer Farmacéutica, Nueva York, Estados Unidos.

\section{Referencias}

1. Abi-said D, Anaissie E, Uzun O, Raad I, Pinzcowski $\mathbf{H}$, Vartivarian S. The epidemiology of hematogenous candidiasis caused by different Candida species. Clin Infect Dis 1997;24:1122-8.

2. Fridkin SK, Jarvis WR. Epidemiology of nosocomial fungal infections. Clin Microbiol Rev 1996;9:499-511.

3. Maenza JR, Keruly JC, Moore RD, Chaisson RE, Merz WG, Gallant JE. Risk factors for fluconazole-resistant candidiasis in human inmunodeficiency virus-infected patients. J Infect Dis 1996;173:219-25.

4. Diassio RB, Benett J, Myers CE. Mode of action of 5flourocytosine. Biochem Pharmacol 1978;27:703-7.

5. Horsburgh CR, Kirkpatrick $\mathbf{C} \mathbf{H}$. Long-term therapy of chronic mucocutaneous candidiasis with ketoconazole: experience with twenty-one patients. Am J Med 1983; 74:23-9.

6. Nguyen MH, Peacock JE, Morris AJ, Tanner DC, Nguyen ML, Snydman DR, et al. The changing face of candidemia: emergence of non-Candida albicans species and antifungal resistence. Am J Med 1996;100: 617-23.

7. Pfaller MA, Jones RN, Doern GV, Sader HS, Messer SA, Houston A, et al. Bloodstream infections due to Candida species: SENTRY antimicrobial surveillance program in North America and Latin America, 1997-1998. Antimicrob Agents Chemother 2000;44:747-51.

8. Rex JH, Rinaldi MG, Pfaller MA. Resistance of Candida species to fluconazole. Antimicrob. Agents Chemother 1995;39:1-8.

9. Revankar SG, Dib OP, Kirkpatrick WR, McAtee RK, Forthergill AW, Rinaldi MG, et al. Clinical evaluation and microbiology of oropharyngeal infection due to fluconazole-resistant Candida in human immunodeficiency virus-infected patients. Clin Infect Dis 1998; 26:960-3.

10. Ng TT, Denning DW. Fluconazole resistance in Candida in patients with AIDS - a therapeutic approach. J Infect 1993;26:117-25.

11. Sangeorzan JA, Bradley SF, He X, Zarins LT, Ridenour GL, Tiballi RN, et al. Epidemiology of oral candidiasis in HIV-infected patients: colonization, infection, treatment and emergence of fluconazole resistance. Am J Med 1994;97:339-46.

12. Troillet N, Durussel C, Bille J, Glauser MP, Chave JP. Correlation between in vitro susceptibility of Candida albicans and fluconazole-resistant oropharyngeal candidiasis in HIV-infected patients. Eur J Clin Microbiol Infect Dis 1993;12:911-5.

13. Bille J, Glauser MP. Evaluation of the susceptibility of pathogenic Candida species to fluconazole. Fluconazole Global Susceptibility Study Group. Eur J Clin Microbiol Infect Dis 1997;16:924-8.

14. St.-Germain G, Laverdière $M$, Pelletier $R$, Bourgault AM, Libman M, Lemieux C, et al. Prevalence and antifungal susceptibility of 442 Candida isolates from blood and other normally sterile sites: results of a 2-year (1996 to 1998) multicenter surveillance study in Quebec, Canadá. J Clin Microbiol 2001;39:949-53.

15. Berrouane YF, Herwaldt LA, Pfaller MA. Trends in antifungal use and epidemiology of nosocomial yeast infections in an university hospital. J Clin Microbiol 1999; 37:531-7.

16. Liebowitz LD, Ashbee HR, Evans EG, Chong Y, Mallatova N, Zaidi M, et al. A two year global evaluation of the susceptibility of Candida species to fluconazole by disk diffusion. Diagn Microbiol Infect Dis 2001;40:2733.

17. Meis J, Petrou M, Bille J, Ellis D, Gibbs D. A global evaluation of the susceptibility of Candida species to fluconazole by disk diffusion. Global Antifungal Surveillance Group. Diag Microbiol Infect Dis 2000;36:215-23.

18. National Committee for Clinical Laboratory Standards. Approved Standard: M2-A6. Performance 
standards for antimicrobial disk susceptibility tests, 6th ed. Wayne, PA: National Committee for Clinical Laboratory Standards; 1997.

19. National Committee for Clinical Laboratory Standards. Reference method for broth dilution antifungal susceptibility testing of yeasts. Approved Standard M27 A NCCLS. Villanova, PA: NCCLS; 1997.

20. Barry AL, Brown SD. Fluconazole disk diffusion procedure for determining susceptibility of Candida species. J Clin Microbiol 1996;34:2154.

21. Bille J. BIOMIC video reading of fluconazole agar disk diffusion susceptibility testing of Candida spp. Clinical isolates compared to NCCLS microbroth dilution. Poster J -120. San Diego, CA: ICAAC; 1998.

22. Rangel-Frausto MS, Wiblin T, Blumberg HM, Saiman L, Patterson J, Rinaldi M, et al. National epidemiology of mycoses survey (NEMIS): variations in rates of bloodstream infections due to Candida species in seven surgical intensive care units and six neonatal intensive care units. Clin Infect Dis 1999;29:253-8.

23. Chang HC, Chang JJ, Chan HS, Huang AH, Wu TL, Lin MC, et al. Evaluation of the Etest for direct antifungal susceptibility testing of yeast in positive blood cultures. J Clin Microbiol 2001;39:1328-33.
24. Rangel-Frausto MS, Wiblin T, Blumberg HM, Saiman L, Patterson J, Rinaldi M, et al. National epidemiology of mycoses survey (NEMIS): variations in rates of bloodstream infections due to Candida species in seven surgical intensive care units and six neonatal intensive care units. Clin Infect Dis 1999;29:253-8.

25. Pfaller MA, Jones RN, Doern GV, Sader HS, Messer SA, Houston A, et al. International surveillance of blood stream infections due to Candida species in the European SENTRY program: species distribution and antifungal susceptibility including the investigational triazole and echinocandin agents. SENTRY participant group (Europe). Diagn Microbiol Infect Dis 1999;35:19-25.

26. Kao AS, Brandt ME, Pruitt WR, Conn LA, Bradley AP, Stephens DS, et al. The epidemiology of candidemia in two United States cities: results of population-based active surveillance. Clin Infect Dis 1999;29:1164-70.

27. Wey SB, Mori M, Pfaller MA, Woolson RF, Wenzel RP. Hospital-acquired candidemia. The attributable mortality and excess length of stay. Arch Intern Med 1988;148: 2642-5.

28. Marina NM, Flynn PM, Rivera GK, Hughes WT. Candida tropicalis and Candida albicans fungemia in children with leukemia. Cancer 1991;68:594-9. 